\title{
Neisseria meningitidis phenotypic markers and septicaemia, disease progress and case-fatality rate of meningococcal disease: a 20-year population- based historical follow-up study in a Danish county
}

\author{
Elise Snitker Jensen, ${ }^{1}$ Henrik C. Schønheyder, ${ }^{2}$ Inga Lind, ${ }^{3}$ \\ Lene Berthelsen, ${ }^{3}$ Bente Nørgård ${ }^{1}$ and Henrik Toft Sørensen ${ }^{1}$ \\ ${ }^{1}$ Department of Clinical Epidemiology, Aalborg and Aarhus University Hospital, Stengade 10, 2nd \\ floor, DK-9000 Aalborg, Denmark \\ ${ }^{2}$ Department of Clinical Microbiology, Aalborg Hospital, DK-9000 Aalborg, Denmark \\ ${ }^{3}$ The Neisseria Unit, Statens Serum Institut, DK-2300 Copenhagen, Denmark
}

Correspondence

Elise Snitker Jensen

esjensen@dadlnet.dk

Received 25 October 2001

Accepted 15 October 2002

\section{INTRODUCTION}

Neisseria meningitidis is an important cause of bacterial meningitis and septicaemia and the high case-fatality rate (CFR) of meningococcal disease (MD) continues to cause public concern. Increases in the incidence rate (IR) of MD have been associated with the introduction of new strains of $N$. meningitidis into the populations of the Czech Republic, Canada, Spain and Greece (Krïzová et al., 1997; Ashton et al., 1991; Berron et al., 1998; Kremastinou et al., 1999). Furthermore, there is evidence that certain serological types may be

Abbreviations: $\mathrm{CFR}$, case-fatality rate; $\mathrm{Cl}$, confidence interval; IR, incidence rate; $\mathrm{MD}$, meningococcal disease; $\mathrm{OR}$, odds ratio; $\mathrm{RR}$, relative risk. associated with an increased CFR (Spanjaard et al., 1987a). Thus, a Dutch study of 536 patients with group BMD reported a higher CFR for serotype $2 b$ than for non-2b (Spanjaard et al., 1987b) and a Norwegian study found a higher CFR for the phenotypes B:15 and C:2a than for any other phenotype (Iversen \& Aavitsland, 1996). These phenotypes are also frequent in Denmark, mainly as B:15:P1.7,16 and C: 2a:P1.2,5 (Lind \& Berthelsen, 1998). During a Danish outbreak, a higher prevalence of septicaemia and an increased CFR were associated with the phenotype B:15:P1.16 (Samuelsson et al., 1992). However, these studies leave open the question of whether associations between certain phenotypes and increased CFR persist over extended periods.

Population genetic studies by use of multilocus enzyme 
electrophoresis (MLEE) (Selander et al., 1986) have shown that the majority of invasive $N$. meningitidis strains worldwide belong to a limited number of clone complexes, of which the so-called ET-5 and ET-37 complexes have played an important role in the northern hemisphere during the last two to three decades (Caugant et al., 1986; Caugant, 1998). Recently, MLEE has been replaced by DNA multilocus sequence typing (MLST) (Maiden et al., 1998; Enright \& Spratt, 1999) as the reference standard for characterization of meningococci. Genetic recombination takes place within $N$. meningitidis, resulting in the emergence of new strains (Maiden, 1993; Maynard Smith et al., 1993). Furthermore, genetic changes occur among strains with any defined phenotype (Scholten et al., 1994), which may be accompanied by altered virulence. However, phenotypic and genotypic characteristics may both be useful in prognosis studies.

Increases in IR and CFR in the late 1980s and early 1990s have previously been reported in North Jutland County, Denmark (Sørensen et al., 1995, 1998). The reasons for these changes are largely unknown, and we hypothesize that either the introduction of new strains or genetic drift in existing ones may have played a role. Our investigations of this hypothesis are presented in two separate county-based studies.

In the present study, we determined phenotypic markers for $98 \%$ of cases confirmed by culture during a 20 -year period (1980-99) and we studied the association between the serological phenotype of the invasive strain and manifestations with septicaemia with or without meningitis, rapidity of disease progress and CFR. We also studied whether changes over time in IR and CFR might be related to the emergence or spread of certain phenotypes.

Genetic changes of meningococcal strains during the study period and the association between genetic markers and CFR have been presented in a separate study (Jensen et al., 2002).

\section{METHODS}

Patients. This population-based historical follow-up study in North Jutland County, Denmark, covered the period 1 January 1980 to 31 December 1999. The county had about 481000 inhabitants on 1 January 1980 and 494000 on 1 January 2000, corresponding to $9 \%$ of the Danish population. The county was served by about 300 general practitioners who provided $24 \mathrm{~h}$ medical care. Hospital treatment was free of charge for all patients. The county was served by six district hospitals and one referral hospital, which also functioned as the district hospital for the city of Aalborg. Two hospitals had paediatric departments.

The North Jutland Meningococcal Research Database contains data on patients who were admitted with MD to any hospital in the county from 1 January 1980 onwards (Sørensen et al., 1995, 1998). The patients were identified from the following sources: (i) the local and national Medical Offices of Health, to which notification is required, (ii) the County Hospital Discharge Registry, (iii) the regional Department of Clinical Microbiology at Aalborg Hospital and (iv) the National Neisseria Reference Laboratory, Statens Serum Institut, Copenhagen.

A diagnosis of $\mathrm{MD}$ was made when at least one of the following criteria was met: (i) isolation of meningococci from blood or cerebrospinal fluid (CSF) specimens; (ii) presence of Gram-negative diplococci in the CSF as determined by direct microscopy; (iii) seroconversion or positive meningococcal antibody test (MAT) $(<3$ on a scale from 0 to 18$)$ combined with clinical findings typical of MD (meningitis and/or septicaemia with typical haemorrhagic rash); or (iv) clinical findings typical of MD (meningitis and/or septicaemia with typical haemorrhagic rash) (Sørensen et al., 1995).

A positive blood culture was used as a surrogate for septicaemia and a positive CSF culture for meningococci. Duration of disease before admission was used as a surrogate for rapidity of disease progress. CFR was defined as the proportion of patients who died during their stay in hospital. Three patients who were dead on arrival at the hospital were included in the study. Hospital and microbiological records were reviewed to ensure that all patients fulfilled the criteria for MD. The following data were collected for each patient: age and sex, date and year of admission, duration of disease before admission $(\leqslant 24 \mathrm{~h}$ or $>24 \mathrm{~h})$, specimen type (CSF and/or blood), bacteriological characteristics of the strain and outcome of disease (death or survival).

A 10-digit personal registration number is given to all residents in Denmark at birth or following immigration. This ID number is used in all health registries, thus making linkage simple and valid.

Microbiological methods. Culture of $N$. meningitidis was performed at the Department of Clinical Microbiology, Aalborg Hospital. CSF specimens were plated on chocolate agar and $10 \%$ horse blood agar (Media Department, Statens Serum Institut) and incubated in $5 \% \mathrm{CO}_{2}$. Three different blood culture systems were used in succession, as described elsewhere (Madsen et al., 1999). Isolates were sent to the Neisseria Unit, Statens Serum Institut, Copenhagen, and stored at $-196^{\circ} \mathrm{C}$. One isolate from each patient was included in the study. Each meningococcal strain was characterized by its serogroup, serotype, serosubtype (Frasch et al., 1985) and sulphonamide resistance.

Serogrouping was performed by co-agglutination using rabbit antisera coated on protein A-rich Staphylococcus aureus cells as carriers (Kronvall, 1972). From July 1999, a latex agglutination test (Slidex méningite-kit 5) was used for determination of serogroups A, B and C, as described by the manufacturer (bioMérieux). Serotyping and serosubtyping were performed by a whole-cell ELISA using mAbs (Lind \& Berthelsen, 1998; Abdillahi \& Poolman, 1987) provided by Rijksinstituut voor Volksgezondheid \& Milieu (RIVM), Bilthoven, The Netherlands. In order to ensure comparability of results, strains obtained before 1990 were retyped with the panel of reagents used after 1990. Susceptibility to sulphonamide was determined by an agar dilution method and resistance was defined as a minimum inhibitory concentration (MIC) $\leqslant 8 \mathrm{mg} \mathrm{l}^{-1}$ (Lind \& Berthelsen, 1998). We defined the phenotype as the combination of serogroup, serotype and serosubtype.

Statistical analysis. Age-standardized IRs were calculated by use of annual population data provided from Statistics Denmark, with the age distribution of the population of North Jutland County 1980-83 as reference.

Phenotypes were divided into three categories: B: 15:P1.7,16, $\mathrm{C}: 2 \mathrm{a}: \mathrm{P} 1.2,5$ and any other phenotype. The manifestation of MD was categorized in two ways: (i) septicaemia alone versus meningitis with or without septicaemia or (ii) septicaemia with or without meningitis versus meningitis alone. The rapidity of disease progress was evaluated on the basis of the duration of symptoms before admission $(\leqslant 24 \mathrm{~h}$ and $>24 \mathrm{~h}$ ). The study period was divided into five 4 -year periods or into two 10 -year periods. The proportions of the three phenotype categories and of cases with negative culture were determined for each 4 -year period.

We obtained contingency tables for the phenotype and the main study variables and $95 \%$ confidence intervals (CIs) for proportions were calculated. Contingency tables were also obtained for the 4-year period and the main study variables. Next, contingency tables were obtained for the main study variables and CFRs. In order to examine the association between phenotype and CFR and to examine the impact of calendar 
period, we fitted a logistic regression model including calendar period as five design variables and age as a continuous variable and calculated odds ratios (ORs) with $95 \%$ CIs. The fit of the model was assessed using the Hosmer-Lemeshow test (Hosmer \& Lemeshow, 1989).

The impact of phenotypes on the CFR during the first and second decade was studied by comparing the prevalences of phenotypes, CFR as related to phenotype and the relative risk (RR) of case fatality for patients with $\mathrm{B}: 15: \mathrm{P} 1,7,16$ and $\mathrm{C}: 2 \mathrm{a}: \mathrm{P} 1.2,5$ compared with any other phenotype. Finally, the difference in CFR between 1980-89 and 199099 was calculated for each phenotype category with $95 \%$ CI.

The study was approved by the Danish Data Protection Agency (journal no. 1994-1200-236).

\section{RESULTS}

During 1980-99, 413 cases of MD were recorded. The overall IR for the entire period was 4.3 per 100000 person-years at risk. The age-standardized IR changed over the five successive 4-year periods: respectively $3 \cdot 6,3 \cdot 9,4 \cdot 3,6 \cdot 3$ and $4 \cdot 8$ per 100000 person-years at risk.

An isolate of $N$. meningitidis was obtained from blood, CSF or both in 320 cases $(77 \%)$. A strain was available for serological characterization from 315 cases (98\%). Serogroup B constituted 226 (72\%) of the strains, serogroup C $75(24 \%)$ and other serogroups $14(4 \%)$. The most frequent serogroup $\mathrm{B}$ phenotype was $\mathrm{B}: 15: \mathrm{P} 1.7,16$ $(n=100,32 \%)$ and the most frequent serogroup $\mathrm{C}$ phenotype was C: $2 \mathrm{a}: \mathrm{P} 1.2,5(n=31,10 \%)$. Apart from B: NT: P1.9 $(n=30,10 \%)$, all other phenotypes each constituted less than $3 \%$ of the strains (Table 1). Resistance to sulphonamides was recorded for all $\mathrm{B}: 15: \mathrm{P} 1.7,16$ strains, for $68 \%$ of the $C: 2 \mathrm{a}: \mathrm{P} 1.2,5$ strains and for $31 \%$ of other strains. The following increases were observed from 1980-83 to 1996-99 in the 315 patients: 7 to $28 \%$ in the proportion who were $\geqslant 30$ years, 9 to $41 \%$ in the proportion with septicaemia without meningitis and 43 to $69 \%$ in the proportion with symptoms for $\leqslant 24 \mathrm{~h}$ before admission. The CFR increased from $1 \cdot 8$ to $17 \%$, with a peak of $21 \%$ during $1988-91$.

Changes over time in the prevalence of cases with phenotypes B : 15: P1.7,16, C:2a:P1.2,5, other phenotypes and without culture-confirmation of the diagnosis are shown in Fig. 1. The number of patients with $\mathrm{B}: 15: \mathrm{P} 1.7,16$ strains doubled during 1992-95, thus contributing markedly to the increase in the total number of cases. Among culture-confirmed cases, the proportion of B : $15:$ P1.7,16 strains increased from $29 \%$ during 1980-83 to 50\% during 1996-99. The prevalence of cases with C : $2 \mathrm{a}:$ P 1.2,5 strains reached a peak of $19 \%$ during 1984-87 and decreased thereafter to $3 \cdot 7 \%$ during 1996-99.

The distribution of patient characteristics and clinical manifestations by phenotype of the invasive strain are shown in Table 2. The median age of patients with $\mathrm{B}: 15: \mathrm{P} 1.7,16$ strains was about 5 years higher than for other MD patients. Septicaemia without meningitis tended to be less common in cases with $\mathrm{B}: 15: \mathrm{P} 1.7,16$ and $\mathrm{C}: 2 \mathrm{a}: \mathrm{P} 1.2,5$ strains. Conversely, a larger proportion of patients with these phenotypes had both meningitis and septicaemia. The CFR was $7 \cdot 3,9 \cdot 5$ and $24.3 \%$ for patients with meningitis only, both meningitis
Table 1. Distribution of serogroups and phenotypes for invasive strains of $N$. meningitidis with a complete phenotype in North Jutland County, Denmark, during 1980-99 $(n=315)$

One isolate from blood or CSF was included per patient. NG, Not grouped; NT, not typed; N-ST, not subtyped.

\begin{tabular}{|c|c|c|}
\hline \multirow[t]{2}{*}{ Phenotype } & \multicolumn{2}{|c|}{ Abundance } \\
\hline & $n$ & $\%$ \\
\hline Serogroup B & 226 & $71 \cdot 7$ \\
\hline $\mathrm{B}: 15: \mathrm{P} 1.7,16$ & 100 & $31 \cdot 7$ \\
\hline $\mathrm{B}: 15: \mathrm{P} 1.7, \mathrm{~B}: 15: \mathrm{P} 1.16$ or $\mathrm{B}: \mathrm{NT}: \mathrm{P} 1.7,16$ & 11 & $3 \cdot 5$ \\
\hline $\mathrm{B}: 4: \mathrm{P} 1.15$ & 9 & $2 \cdot 9$ \\
\hline B : $14: \mathrm{P} 1.7,16$ & 9 & $2 \cdot 9$ \\
\hline B : $16: \mathrm{P} 1.16$ & 9 & $2 \cdot 9$ \\
\hline $\mathrm{B}: \mathrm{NT}: \mathrm{N}-\mathrm{ST}$ & 8 & $2 \cdot 5$ \\
\hline B : NT : P1.9 & 30 & $9 \cdot 5$ \\
\hline Other serogroup B ( $\leqslant 7$ of each) & 50 & $15 \cdot 9$ \\
\hline Serogroup C & 75 & $23 \cdot 8$ \\
\hline $\mathrm{C}: 2 \mathrm{a}: \mathrm{P} 1.2,5$ & 31 & $9 \cdot 8$ \\
\hline $\mathrm{C}: 2 \mathrm{a}: \mathrm{P} 1.2, \mathrm{C}: 2 \mathrm{a}: \mathrm{N}-\mathrm{ST}$ or $\mathrm{C}: \mathrm{NT}: \mathrm{P} 1.2,5$ & 17 & $5 \cdot 4$ \\
\hline $\mathrm{C}: 2 \mathrm{~b}: \mathrm{P} 1.2,5$ & 9 & $2 \cdot 9$ \\
\hline Other serogroup C ( $\leqslant 4$ of each) & 18 & $5 \cdot 7$ \\
\hline Serogroup A, W135, Y or NG* & 14 & $4 \cdot 4$ \\
\hline
\end{tabular}

*Various phenotypes.

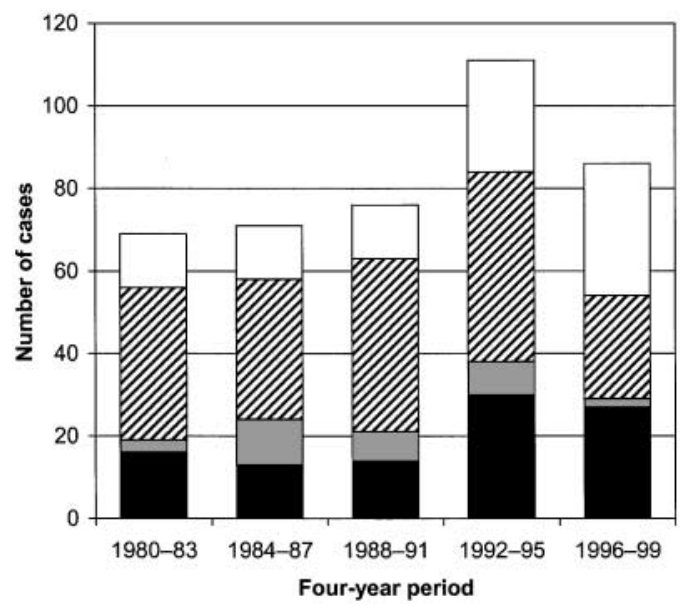

Fig. 1. Changes over time in the distribution of phenotypes of $N$. meningitidis obtained from blood or CSF in patients with MD and of culture-negative MD cases in North Jutland County, Denmark, 198099. Numbers of cases of $\mathrm{B}: 15: \mathrm{P} 1.7,16$ (filled bars), C : 2a:P1.2,5 (shaded bars) or other phenotypes (hatched bars) and cases that were culture-negative or where the isolate was lost (open bars) are shown.

and septicaemia and septicaemia only, respectively (data not shown). No association was found between phenotype and rapid disease progress. The CFR was $14.7 \%$ for patients with duration of illness $\leqslant 24 \mathrm{~h}$ and $7.9 \%$ for patients with duration of illness $>24 \mathrm{~h}$ (data not shown). 
Table 2. Characteristics of 315 patients with culture-confirmed MD in North Jutland County, Denmark 1980-99 by phenotypic markers $95 \%$ CIs for percentages are given in parentheses.

\begin{tabular}{|c|c|c|c|c|}
\hline Characteristic & $\begin{array}{c}\text { B }: 15: P 1.7,16 \\
\quad(n=100)\end{array}$ & $\begin{array}{c}\mathrm{C}: 2 \mathrm{a}: \mathrm{P} 1.2,5 \\
(n=31)\end{array}$ & $\begin{array}{l}\text { Other (ref.) } \\
(n=184)\end{array}$ & Total $(n=315)$ \\
\hline \multicolumn{5}{|l|}{ Age (years): } \\
\hline 1st-3rd quartiles & $3-18$ & $2-19$ & $2-21$ & $2-19$ \\
\hline Sex (male, $n / \%)$ & $52 / 52(42-62)$ & $17 / 55(36-73)$ & $100 / 54(47-62)$ & $169 / 54(48-59)$ \\
\hline Septicaemia and meningitis $(n / \%)$ & $42 / 42(32-52)$ & $14 / 45(27-64)$ & $39 / 21(16-28)$ & $95 / 30(25-36)$ \\
\hline Meningitis without septicaemia $(n / \%)$ & $41 / 41(31-51)$ & $11 / 36(19-55)$ & $98 / 53(46-61)$ & $150 / 48(42-53)$ \\
\hline Pre-admission duration of disease ${ }^{*}(\leqslant 24 \mathrm{~h}, n / \%)$ & $60 / 61(50-70)$ & $15 / 50(31-69)$ & $95 / 53(45-60)$ & $170 / 55(49-61)$ \\
\hline Death $(n / \%)$ & $15 / 15(9-24)$ & $7 / 23(10-41)$ & $15 / 8(5-13)$ & $37 / 12(8-16)$ \\
\hline
\end{tabular}

* Based on 309 patients; information was missing for six patients.

The CFR was higher for patients with B: 15:P1.7,16 and $\mathrm{C}: 2 \mathrm{a}: \mathrm{P} 1.2,5$ strains than for patients with other phenotypes. In addition, there was an association between calendar period and CFR, with an increased CFR during 1988-99, reaching a maximum during 1988-91 (Table 3).

During 1990-99, B : 15 : P1.7,16 constituted a larger proportion of the strains than during the previous decade and the CFR for patients with this phenotype increased from 7.9 to $19 \cdot 4 \%$. Despite a fourfold increase in the number of fatalities among patients with B : 15 : P1.7,16 strains during 1990-99, there was less variation of CFR between phenotype categories because the CFR also increased from $2 \cdot 3$ to $13.5 \%$ for patients with other phenotypes. During 1990-99, C:2a: P1.2,5 constituted a smaller proportion of the strains than during the previous decade. Thus, despite a high CFR ( $>20 \%$ ) throughout the study period, this phenotype contributed less to the overall case fatality during the second decade than during the first (Table 4).

\section{DISCUSSION}

The prevalence of MD caused by $\mathrm{B}: 15: \mathrm{P} 1.7,16$ strains increased markedly during the 20 years under study. The meningococcal phenotypes $\mathrm{B}: 15: \mathrm{P} 1.7,16$ and $\mathrm{C}: 2 \mathrm{a}: \mathrm{P} 1.2,5$ were associated with an increased CFR compared with that of any other phenotype. Unexpectedly, we found septicaemia without meningitidis to be relatively rare for phenotypes B : 15 : P1.7,16 and C : 2a : P1.2,5 and no association between

Table 3. Crude and adjusted ORs of CFR related to phenotype and calendar period for 315 patients with culture-confirmed MD in North Jutland County, Denmark, during 1980-99

Hosmer-Lemeshow test statistics: $\chi^{2}=5 \cdot 8$, d.f. $=8, P=0 \cdot 7$. Values for ORs have $95 \%$ CIs in parentheses.

\begin{tabular}{|lrccc|}
\hline Category & $\begin{array}{c}\text { Fatal cases } \\
(\boldsymbol{n} / \text { total })\end{array}$ & CFR $(\%)$ & Crude OR & Adjusted OR $^{*}$ \\
\hline Phenotype & & & & \\
Other & $15 / 184$ & $8 \cdot 2$ & $1 \cdot 0$ & $1 \cdot 0$ \\
B: $15:$ P1.7,16 & $15 / 100$ & $15 \cdot 0$ & $2 \cdot 0(0 \cdot 9-4 \cdot 3)$ & $2 \cdot 8(1 \cdot 2-18 \cdot 5)$ \\
C:2a : P1.2,5 & $7 / 31$ & $22 \cdot 6$ & $3 \cdot 3(1 \cdot 2-8 \cdot 9)$ & $5 \cdot 2(1 \cdot 6-16 \cdot 4)$ \\
Calendar year & & & & $1 \cdot 0$ \\
$1980-83$ & $1 / 56$ & $1 \cdot 8$ & $1 \cdot 0$ & $1 \cdot 7(0 \cdot 2-18 \cdot 5)$ \\
$1984-87$ & $3 / 58$ & $5 \cdot 2$ & $3 \cdot 0(0 \cdot 3-29 \cdot 6)$ & $5 \cdot 3(0 \cdot 6-45 \cdot 6)$ \\
$1988-91$ & $13 / 63$ & $20 \cdot 6$ & $14 \cdot 3(1 \cdot 8-112 \cdot 8)$ & $12 \cdot 5(1 \cdot 5-106 \cdot 6)$ \\
$1992-95$ & $11 / 84$ & $13 \cdot 1$ & $8 \cdot 3(1 \cdot 0-65 \cdot 8)$ & $5 \cdot 0(0 \cdot 7-53 \cdot 2)$ \\
$1996-99$ & $9 / 54$ & $16 \cdot 7$ & $11 \cdot 0(1 \cdot 3-89 \cdot 7)$ & \\
\hline
\end{tabular}

*Adjusted for age as a continuous variable. 
phenotype and rapid disease progress. The increased prevalence of patients with $\mathrm{B}: 15: \mathrm{P} 1.7,16$ strains and an increased CFR among these patients contributed to the increased overall CFR during 1990-99 compared with 1980-89. In addition, increased CFR was associated with calendar period.

The major strength of the present study derives from its population-based design, with complete registration of MD cases and complete follow-up during 20 years in a welldefined population with a uniformly organized health-care system (Sørensen et al., 1995). A limitation of our study was the limited statistical precision for subgroup analysis. Another limitation was the exclusion of $23 \%$ of the patients because the diagnosis was not confirmed by culture. This may have led to selection bias of the overall association between phenotype and CFR. The direction will depend on whether the strength of the association in patients with culture-confirmed MD differs from the unknown association in excluded patients. We are aware that the heterogeneous group of phenotypes used as reference may include phenotypes with distinctly different associations with CFR. However, we did not detect any variation between the different phenotypes (data not shown), though the small numbers should be taken into consideration.

The specificity of the antibodies used for serotyping and serosubtyping is considered to be $100 \%$ (Poolman et al., 1995). Thus, misclassification of strains that have been assigned a complete phenotype can be excluded with confidence. Non-typable and non-subtypable strains may differ from the fully typable strains in respects other than typability, which is why these strains were assigned to the reference group.

The categorization of duration of illness before admission was intended to distinguish a fulminant course, which is a well-known risk factor for fatal outcome, from the more protracted courses (Van Deuren et al., 2000; Sinclair et al., 1987). Our choice of a positive blood culture as a surrogate for septicaemia is in accordance with the definition of septicaemia, which includes a positive blood culture combined with overt clinical signs of infection (Effersøe \& Nielsen, 1986). We deem the latter to have been verified by the fact that the patient was evaluated clinically before sampling of blood for culture. MD evolves to meningitis through haematogenous dissemination, and a positive CSF culture may therefore represent a distinct category of patients who have survived the initial bacteraemic stage and might thus have a better prognosis than patients with positive culture of blood only (Van Deuren et al., 2000). Disease progress and manifestation of disease (septicaemia alone, meningitis and septicaemia or meningitis alone) may be regarded as intermediate steps in the process of infection and, as such, they were not included as potential confounders in the logistic regression model.

During 1980-85, the mean annual IR of notified cases of MD in Denmark was 3.4 per 100000 person-years at risk. In 1986, the IR increased by $60 \%$ and, during 1986-88, the mean annual IR was 5.4 per 100000 person-years at risk 
(Samuelsson et al., 1991). In North Jutland County, a similar increase occurred with a delay of about 5 years. Reliable data on CFR for all of Denmark are available from 1988 onwards and, during 1988-91, 1992-95 and 1996-99, the CFR was respectively $9 \cdot 6,8 \cdot 1$ and $7 \cdot 8 \%$ (for North Jutland CFRs, see Table 3). We have no explanation for this difference. Since 1993, B : $15:$ P1.7,16 and C: $2 \mathrm{a}:$ P1.2,5 have respectively been confirmed to be the predominant phenotypes among serogroup B and C strains (L. Berthelsen, unpublished observations; Lind \& Berthelsen, 1998). North Jutland County is an important holiday resort, with tourists from many European countries including many teenagers. This should provide opportunities for the introduction of novel meningococcal strains. However, we found meningococcal phenotypes to be stable over an extended period of time.

It is not clear whether the most relevant markers for the identification of virulent strains are phenotypic or genotypic. In a separate study (Jensen et al., 2002), we selected 181 strains for further characterization by MLEE and ribotyping. We identified two distinctive clone complexes among serogroup B strains (including 92 of the $100 \mathrm{~B}: 15:$ P $1.7,16$ strains) and one distinctive clone complex among serogroup $\mathrm{C}$ strains (including 30 of the $31 \mathrm{C}: 2 \mathrm{a}: \mathrm{P} 1.2,5$ strains). Each of the three clone complexes was associated with an increased CFR (13-20\%) compared with any other strain (CFR 3\%) (Jensen et al., 2002). However, the CFRs for phenotypes $\mathrm{B}: 15: \mathrm{P} 1.7,16$ and $\mathrm{C}: 2 \mathrm{a}: \mathrm{P} 1.2,5$ tended to be higher than the CFRs for the three clonal complexes. This points to the phenotypes being relevant surrogate markers for determinants of virulence of meningococci.

In accordance with our finding, a high CFR was reported in patients with $\mathrm{C}: 2 \mathrm{a}: \mathrm{P} 1.2,5$ strains in the Czech Republic and, in Norway, CFR was increased in patients with B : 15 or C:2a strains (Krïzová et al., 1997; Iversen \& Aavitsland, 1996). However, the Norwegian data were based on notified cases only and the analysis included only 105 patients admitted during one year, 1994. In contrast to the strains from the Czech Republic, we found that the CFR associated with C:2a:P1.2,5 infection remained high $(>20 \%)$ over a 20 year period, whereas the CFR associated with $\mathrm{B}: 15: \mathrm{P} 1.7,16$ increased from an intermediate to a high level. The Norwegian study found no difference in clinical manifestations between serogroup $B$ and serogroup $C \mathrm{MD}$, but it is not clear whether this applied to $B: 15$ and $C: 2 a$ MD. Furthermore, the classification of septicaemia versus meningitis was based on clinical criteria and is thus not directly comparable with ours. In a previous Danish study, the increased CFR associated with $\mathrm{B}: 15: \mathrm{P} 1.16$ was related to a high proportion of patients with septicaemia, but it is noteworthy that, in septicaemic patients, the CFR for B : 15 : P1.16 did not differ from that of other phenotypes (Samuelsson et al., 1992). Furthermore, the latter study related to an outbreak situation, whereas our study covered a long period of high endemicity. This setting resembled the situation in Norway, where $\mathrm{B}: 15: \mathrm{P} 1.7,16$ (belonging to the ET-5 complex) has been predominant for more than two decades. In contrast to other European countries, in which increases in IR have been related to C :2a : P1.2,5 (Krïzová et al., 1997; Kremastinou et al., 1999; Connolly \& Noah, 1999), the occurrence of this phenotype actually decreased in North Jutland County during 1990-99.

The increased prevalence of $\mathrm{B}: 15: \mathrm{P} 1.7,16$ cases during 1992-99 could be a reflection of increased virulence or waning population immunity. Taha et al. (2001) recently described a mutation in the DNA region encoding one of the epitopes determining the dual serosubtype in $\mathrm{B}: 15: \mathrm{P} 1.7,16$ meningococci. As a result, the epitope was not recognized by the bactericidal serum antibodies that were prevalent in the population (Taha et al., 2001). Thus, underlying genetic changes might explain a sudden decrease in population immunity against $\mathrm{B}: 15: \mathrm{P} 1.7,16$ despite frequent occurrence for more than a decade. Theoretically, evasion of bactericidal antibodies, as described above, may lead not only to increased incidence of disease but also to increased CFR among patients infected with the mutated strain. However, we did not detect any genotypic change of $\mathrm{B}: 15: \mathrm{P} 1.7,16$ strains that correlates with the increases in IR and CFR (Jensen et al., 2002).

The lack of association in the present study between phenotype and septicaemia alone and between phenotype and rapid disease progress is surprising (Van Deuren et al., 2000; Sinclair et al., 1987); it suggests that these factors may be related to host factors rather than to the pathogen itself. Our finding of an association between calendar period and prognosis, which was independent of meningococcal phenotype and age of the patient, indicates that one or more hitherto unknown factors contributed to the observed trend in CFR. The increases in the proportions of patients with septicaemia alone and of patients with short pre-admission duration of disease are likely to have contributed to the increased CFR, but remain in themselves unexplained. This underlines the need for further research into the pathogenesis of $\mathrm{MD}$, including the possible impact of meningococcal phenotypic and genotypic characteristics.

\section{ACKNOWLEDGEMENTS}

This work was supported by grants from the Ville Heise Foundation (Ville Heises Legat), the Obel Family Foundation (Det Obelske Familiefond), the Danish Medical Research Council (grant no. 9700677), the Research Foundation at Hjørring Hospital, founded by Niels Jensen (Forskningsfonden ved Hjørring Sygehus stiftet af Niels Jensen), the Christmas Lottery of Aalborg Diocese (Aalborg Stifts Julelotteri), the Health Insurance Foundation (Sygekassernes Helsefond), the North Jutland County Research Foundation (Nordjyllands Amts Forskningsfond) and the PhD programme at Aalborg Hospital. We thank Ms Liselotte Winther Sørensen and the late Ms Inger Lindhardt for practical assistance.

\section{REFERENCES}

Abdillahi, H. \& Poolman, J. T. (1987). Whole-cell ELISA for typing Neisseria meningitidis with monoclonal antibodies. FEMS Microbiol Lett 48, 367-371.

Ashton, F. E., Ryan, J. A., Borczyk, A., Caugant, D. A., Mancino, L. \& 
Huang, D. (1991). Emergence of a virulent clone of Neisseria meningitidis serotype $2 \mathrm{a}$ that is associated with meningococcal group $\mathrm{C}$ disease in Canada. J Clin Microbiol 29, 2489-2493.

Berron, S., De La Fuente, L., Martin, E. \& Vázquez, J. A. (1998). Increasing incidence of meningococcal disease in Spain associated with a new variant of serogroup C. Eur J Clin Microbiol Infect Dis 17, 85-89.

Caugant, D. A. (1998). Population genetics and molecular epidemiology of Neisseria meningitidis. APMIS 106, 505-525.

Caugant, D. A., Frøholm, L. O., Bøvre, K., Holten, E., Frasch, C. E., Mocca, L. F., Zollinger, W. D. \& Selander, R. K. (1986). Intercontinental spread of a genetically distinctive complex of clones of Neisseria meningitidis causing epidemic disease. Proc Natl Acad Sci U S A 83, 4927-4931.

Connolly, M. \& Noah, N. D. (1999). Is group C meningococcal disease increasing in Europe? A report of surveillance of meningococcal infection in Europe 1993-6. European Meningitis Surveillance Group. Epidemiol Infect 122, 41-49.

Effersøe, P. \& Nielsen, J. O. (1986). Infektionssygdomme. In Medicinsk Kompendium, pp. 418-422. Edited by J. H. Thaysen, L. K. Christensen \& I. Lorentsen. Copenhagen: Nyt Nordisk Forlag Arnold Busck.

Enright, M. C. \& Spratt, B. G. (1999). Multilocus sequence typing. Trends Microbiol 7, 482-487.

Frasch, C. E., Zollinger, W. D. \& Poolman, J. T. (1985). Serotype antigens of Neisseria meningitidis and a proposed scheme for designation of serotypes. Rev Infect Dis 7, 504-510.

Hosmer, D. W. \& Lemeshow, S. (1989). Applied Logistic Regression. New York: Wiley.

Iversen, B. G. \& Aavitsland, P. (1996). Meningococcal disease in Norway 1992-1995. Epidemiology and fatality. Scand J Infect Dis 28, 253-259.

Jensen, E. S., Berthelsen, L., Lind, I., Fussing, V., Sørensen, H. T. \& Schønheyder, H. C. (2002). Period prevalence and case-fatality rate associated with distinctive clone complexes of Neisseria meningitidis serogroups B and C. Eur J Clin Microbiol Infect Dis 21, 506-512.

Kremastinou, J., Tzanakaki, G., Kansouzidou, A. \& 8 other authors (1999). Recent emergence of serogroup $C$ meningococcal disease in Greece. FEMS Immunol Med Microbiol 23, 49-55.

Krïzová, P., Musilek, M. \& Kalmusová, J. (1997). Development of the epidemiological situation in invasive meningococcal disease in the Czech Republic caused by emerging Neisseria meningitidis clone ET-15/ 37. Cent Eur J Public Health 5, 214-218.

Kronvall, G. (1972). A rapid slide-agglutination method for typing pneumococci by means of specific antibody adsorbed to protein Acontaining staphylococci. J Med Microbiol 6, 187-190.

Lind, I. \& Berthelsen, L. (1998). Meningokoksygdom 1993-1996. Nyt Mikrobiol 46, 5-10.

Madsen, K. M., Schønheyder, H. C., Kristensen, B. \& Sørensen, H. T. (1999). Secular trends in incidence and mortality of bacteraemia in a Danish county 1981-1994. APMIS 107, 346-352.
Maiden, M. C. (1993). Population genetics of a transformable bacterium: the influence of horizontal genetic exchange on the biology of Neisseria meningitidis. FEMS Microbiol Lett 112, 243-250.

Maiden, M. C., Bygraves, J. A., Feil, E. \& 10 other authors (1998). Multilocus sequence typing: a portable approach to the identification of clones within populations of pathogenic microorganisms. Proc Natl Acad Sci U S A 95, 3140-3145.

Maynard Smith, J., Smith, N. H., O'Rourke, M. \& Spratt, B. G. (1993). How clonal are bacteria? Proc Natl Acad Sci U S A 90, 4384-4388.

Poolman, J. T., Kriz-Kuzemenska, P., Ashton, F. \& 9 other authors (1995). Serotypes and subtypes of Neisseria meningitidis: results of an international study comparing sensitivities and specificities of monoclonal antibodies. Clin Diagn Lab Immunol 2, 69-72.

Samuelsson, S., Gustavsen, S. \& Rønne, T. (1991). Epidemiology of meningococcal disease in Denmark 1980-1988. Scand J Infect Dis 23, 723-730.

Samuelsson, S., Ege, P., Berthelsen, L. \& Lind, I. (1992). An outbreak of serogroup B:15:P1.16 meningococcal disease, Frederiksborg County, Denmark, 1987-9. Epidemiol Infect 108, 19-30.

Scholten, R. J., Poolman, J. T., Valkenburg, H. A., Bijlmer, H. A., Dankert, J. \& Caugant, D. A. (1994). Phenotypic and genotypic changes in a new clone complex of Neisseria meningitidis causing disease in The Netherlands, 1958-1990. J Infect Dis 169, 673-676.

Selander, R. K., Caugant, D. A., Ochman, H., Musser, J. M., Gilmour, M. N. \& Whittam, T. S. (1986). Methods of multilocus enzyme electrophoresis for bacterial population genetics and systematics. Appl Environ Microbiol 51, 873-884.

Sinclair, J. F., Skeoch, C. H. \& Hallworth, D. (1987). Prognosis of meningococcal septicaemia. Lancet ii, 38.

Sørensen, H. T., Hansen, I., Ejlersen, E., Schønheyder, H. C., Hamburger, H. \& Sabroe, S. (1995). Identification of cases of meningococcal disease: data quality in two Danish population-based information systems during a 14-year period. Int J Risk Saf Med 7, 179-189.

Sørensen, H. T., Steffensen, F. H., Schønheyder, H. C., Nielsen, G. L., Hansen, I., Madsen, K. M. \& Hamburger, H. (1998). Trend in incidence and case fatality of meningococcal disease over 16 years in northern Denmark. Eur J Clin Microbiol Infect Dis 17, 690-694.

Spanjaard, L., Bol, P., De Marie, S. \& Zanen, H. C. (1987a). Association of meningococcal serogroups with the course of disease in the Netherlands, 1959-83. Bull World Health Organ 65, 861-868.

Spanjaard, L., Bol, P., De Marie, S. \& Zanen, H. C. (1987b). Association of meningococcal serotypes with the course of disease: serotypes 2a and $2 \mathrm{~b}$ in the Netherlands, 1959-1981. J Infect Dis 155, 277-282.

Taha, M. K., Bichier, E., Perrocheau, A. \& Alonso, J. M. (2001). Circumvention of herd immunity during an outbreak of meningococcal disease could be correlated to escape mutation in the porA gene of Neisseria meningitidis. Infect Immun 69, 1971-1973.

Van Deuren, M., Brandtzaeg, P. \& Van der Meer, J. W. M. (2000). Update on meningococcal disease with emphasis on pathogenesis and clinical management. Clin Microbiol Rev 13, 144-166. 